\title{
Gingivitis and Associated Factors Among Children in Boxes Toddlers in Senegal
}

\author{
Daouda Cisse $^{1}$, Aida Kanouté ${ }^{1}$, Massamba Diouf ${ }^{1}$, Sylvie Azogui-Levy ${ }^{2}$, Boubacar S. Dankoko ${ }^{3}$, \\ Astou Mbengue Niang ${ }^{4}$, Mbathio Diop ${ }^{1}$, Cheikh M. Mbacké Lo ${ }^{1}$, Daouda Faye ${ }^{1}$ \\ ${ }^{1}$ Public Health Service, Department of Dentistry, Faculty of Medicine, Pharmacy and Dentistry, University Cheikh Anta Diop, Dakar, \\ Senegal \\ ${ }^{2}$ Dental Public Health Service, and Educations and Health Practices Laboratory, Paris-Diderot University, University Paris 13, Paris, France \\ ${ }^{3}$ Public Health and Preventive Medicine Service, Department of Medicine, Faculty of Medicine, Pharmacy and Dentistry, University Cheikh \\ Anta Diop, Dakar, Senegal \\ ${ }^{4}$ Free-Lancer, Dakar, Senegal
}

Email address:

daoudacisse@hotmail.com (D. Cisse)

\section{To cite this article:}

Daouda Cisse, Aida Kanouté, Massamba Diouf, Sylvie Azogui-Levy, Boubacar S. Dankoko, Astou Mbengue Niang, Mbathio Diop, Cheikh M. Mbacké Lo, Daouda Faye. Gingivitis and Associated Factors Among Children in Boxes Toddlers in Senegal. Science Journal of Public Health. Vol. 4, No. 3, 2016, pp. 259-264. doi: 10.11648/j.sjph.20160403.25

Received: April 29, 2016; Accepted: May 10, 2016; Published: May 26, 2016

\begin{abstract}
The aim of this study was to determine the prevalence of gingivitis and the factors associated in boxes toddlers among 3-6 year-old preschool children in 3 regions in Senegal. This was a descriptive and cross sectional study covering 984 preschool children aged 3 to 6 years and attending boxes toddlers in the regions of Dakar (urban area), Saint-Louis (semi-urban and urban areas) and Kaolack (semi-urban and rural areas). They were selected through both a cluster sampling and a stratified sampling and the clusters were drawn at random. These children obtained parental permission to participate in the survey. The socio-demographic data, the gingivitis status and the parental knowledge on oral preventive methods applied to children, were collected. The data was analysed using SPSS Version 19 software with a significance level of 5\%. In total 901 children were examined: 299 in Dakar, 302 in Saint-Louis and 300 in Kaolack with respective completeness rate of 93\%, 91\% and 90\%. Girls constituted $56.6 \%$ of the sample; children aged six were less represented (10.7\%), while children of non-salaried parents constituted $77.3 \%$ of the sample. Fourteen percent of the children had an inflamed gingiva. The distribution of gingivitis according to socio-demographic data does not show a significant association. Depending on the region, gingivitis differently distributed $(p<0.01)$. Between Dakar and Saint-Louis $(p<0.01)$ and between Dakar and Kaolack $(p<0.02)$, the differences were significant. However between Saint-Louis and Kaolack, there was not a significant difference ( $p>0.90)$. Gingivitis is significantly associated with the frequency of brushing $(\mathrm{p}=0.03)$, it is not significantly associated with visiting the dentist $(\mathrm{p}>0.20)$. These results suggest the importance of hygiene and perhaps the parent oral health literacy in the prevention of this infection often overlooked because painless most of the time. To avoid damage as noma more prevalent in rural area, health officials may develop integrated community programs against gingivitis in boxes toddlers in Senegal.
\end{abstract}

Keywords: Gingivitis, Box Toddlers, 3-6 Year-Old Preschool Children, Urban Area, Rural Area, Senegal

\section{Introduction}

According to WHO, in the early twenty-first century, children are among the most vulnerable [1]. Their health is a priority for public health, especially in developing countries where infectious diseases still claim many victims [2]. However because of these infectious diseases, health authorities relegate the oral pathologies in a second place, while these oral pathologies, including gingivitis, are a real health problem given their impact on health, development and quality of life among children [3]. The child's periodontal, seat of this gingivitis, remains a little known structure. Indeed a rigid description of the child's periodontal is difficult because of its permanent changes between the 
eruption of primary teeth, their exfoliation followed by the appearance of permanent teeth and their implementation occlusion. Because of its immature character, periodontal temporary tooth has a certain fragility and offers little resistance to infection during the different phases of the teeth $[4,5]$. Gingivitis is acute or chronic inflammatory damage characterized by redness, oedema and bleeding on probing, in relation to the presence of bacterial plaque, medications, certain systemic diseases (malnutrition, type I diabetes, trisomy 21, AIDS... etc.). Gingivitis is a good hygiene and malnutrition indicator in children. When treated, gingivitis is reversible with no permanent damage. Untreated cases may lead to a more complex and destructive entity as noma in Africa [6-8]. In order to have the current epidemiological data that could contribute to the implementation of oral health prevention program, we conducted a cross-sectional study on the oral health of preschool children in three regions in Senegal in urban, semi-urban and rural areas. This work aimed at studying the prevalence of gingivitis and factors associated in boxes toddlers.

\section{Material and Method}

\subsection{Framework and Type of Study}

In Senegal, since 2002, the establishment of the hut toddlers participates in the management of preschool children as well as in education, nutrition and health [9-11]. This study was conducted in boxes toddlers located in different geographical areas in three regions in Senegal: urban area in the region of Dakar, urban and semi-urban areas in the region of Saint- Louis, and semi-urban and rural areas in the region of Kaolack. These regions were selected primarily for some geographical reasons like the WHO recommendations for nationwide surveys [12]. This was a cross-sectional study describing the presence of gingivitis and collecting sociodemographic characteristics and preventive methods in children boxes toddlers.

\subsection{Studied Population, Sampling and Sample Size}

The studied population consisted of 3 to 6 year-old children in boxes toddlers. All the selected children, at the time of the survey, were eligible for the study. Both stratified sampling and cluster sampling were employed. Indeed the three regions considered as strata had different geographical features. The boxes toddlers in each region formed a set of clusters, each box corresponding to a cluster. Children of all boxes toddlers drawn from a based sampling, formed the sample size set at 300 in each region, taking into account WHO recommendations [12]. Because drawn clusters (boxes) do not have the same number of children, expected size could be obtained either slightly below or above the size set of 300 per region. Thus the size of the total sample for the 3 regions was increased to 984 (Table 1).

Table 1. Distribution of Boys and Girls in Boxes Toddlers by Region and Area.

\begin{tabular}{|c|c|c|c|c|c|}
\hline Region & Area & Name of the box toddlers & Boys & Girls & Total \\
\hline \multirow{3}{*}{ Dakar } & \multirow{3}{*}{ Urban } & HLM & 66 & 70 & \multirow{3}{*}{321} \\
\hline & & Ouest foire & 55 & 59 & \\
\hline & & Douta Seck & 36 & 35 & \\
\hline \multirow{4}{*}{ Saint-Louis } & \multirow{4}{*}{ Urban and semi-urban } & Pikine & 56 & 63 & \multirow{4}{*}{331} \\
\hline & & Ndaté Yalla & 49 & 40 & \\
\hline & & Darou & 35 & 40 & \\
\hline & & Zone Nord & 23 & 25 & \\
\hline \multirow{6}{*}{ Kaolack } & \multirow{6}{*}{ Semi-urban and rural } & Wack Ngouna & 40 & 45 & \multirow{6}{*}{332} \\
\hline & & Koutal & 55 & 26 & \\
\hline & & Guinguinéo & 24 & 27 & \\
\hline & & Khelcom & 13 & 6 & \\
\hline & & Kawsara Fall & 9 & 12 & \\
\hline & & Sibassor & 34 & 41 & \\
\hline Total & & & 495 & 489 & 984 \\
\hline
\end{tabular}

\subsection{Data Collection}

The data collection took place from the $17^{\text {th }}$ of February to $16^{\text {th }}$ of May 2014. The questionnaire had two sections; the first section contained general information about the child (age, sex, place of residence, occupation of parents) and issues related to parental knowledge on oral preventive methods applied to children (frequency of brushing, visit to the dentist). The second section focused on the child's oral health status (gingival status). This questionnaire was pretested in Dakar among children in box toddler out of the boxes toddlers selected. Then it was distributed either directly to parents who came to pick up their children after school, or indirectly by having teachers put questions to be answered in the children's bags. The child's mouth was examined in daylight when the parent had authorized him or her to participate in the survey. Using a mirror and a periodontal WHO probe, evaluation of gingival status was conducted by a student in his 6th year of study in dental surgery, already calibrated to techniques of collecting data; she was helped by the instructors or monitors of boxes toddlers. This assessment of the gingival condition was to classify children into 4 categories according to the degree of affection of the gingival margin: 0 : healthy gingiva; 1 : slight inflammation; 2: moderate inflammation (bleeding at the 
touch of the marginal gingiva with the edge of dental mirror); 3: severe inflammation (spontaneous bleeding). This approach makes it more reliable in terms of reproductibility of gingival index [13]. Once the above mentioned questionnaire was completed, it was recovered, collected and held by teachers of the boxes toddlers.

\subsection{Data Analysis}

The analysis included a total of 901 out of 984 children aged from 3 to 6 . Eighty three questionnaires were unusable. First, univariate analysis was conducted on the sociodemographic variables and variables of gingivitis. Then, a bivariate analysis (chi-square test) associated gingivitis variables on the one hand and socio-demographic and preventive methods data, on the other. To make it more convenient bivariate analyzes, some variables, such as gingival status with more than 3 terms, were recoded into 2 terms. Only the age variable was analyzed with 4 modalities. The significance of the analysis by the SPSS Version 19 software was set at $5 \%$.

\subsection{Organizational and Ethical Considerations}

An authorization with a request for information was made to the national agency for early childhood and box toddlers "Agence Nationale de la Petite Enfance et de la Case des Tout-Petits", concerning the number and location of boxes toddlers in the selected regions. In addition, an information sheet was given to parents for their consent. Thereafter, a circular authorization, signed by Mrs. the Executive director of the agency, was presented to all managers or directors of boxes toddlers before the collection of information was done by appointment, according to a given schedule.

\section{Results}

In total 901 children were examined: 299 in Dakar, 302 in Saint-Louis and 300 in Kaolack with respective completeness rate of $93 \%, 91 \%$ and $90 \%$.

\subsection{Sociodemographic Data}

From the total sample, there were $56.6 \%$ of girls; children aged 6 were less represented $(10.7 \%=(38$ children in Dakar +9 children in Saint-Louis +49 children in Kaolack)/901) while children of non-salaried parents constituted $77.3 \%$ of the sample. Figure 1 describes the frequency of sociodemographic data by region in the sample.

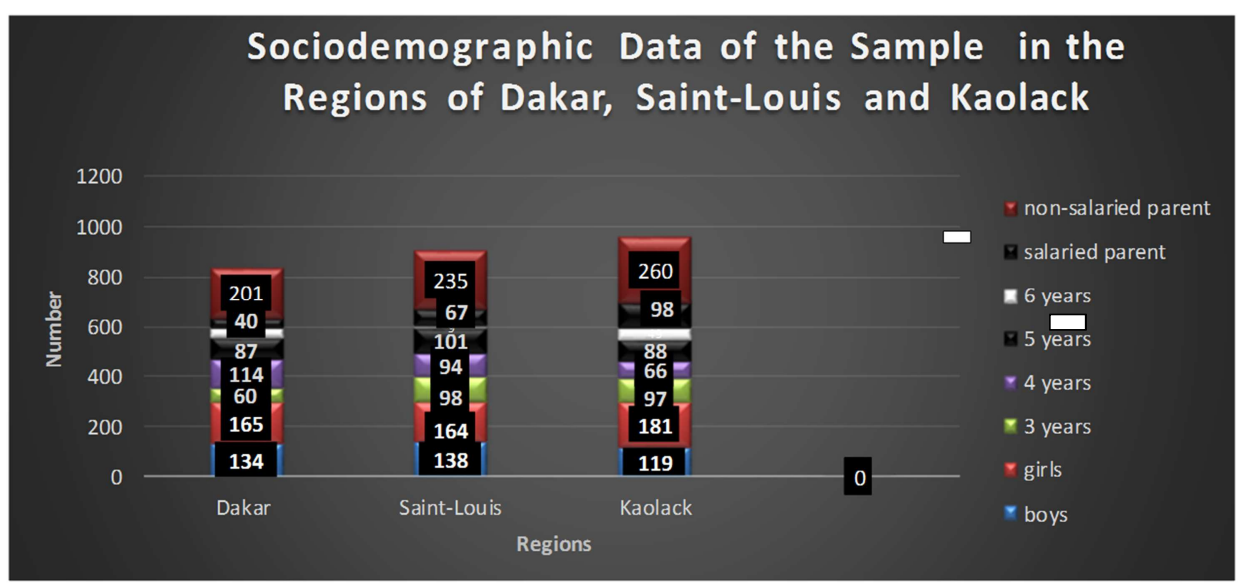

Figure 1. Distribution of Socio-demographic Data by Region in the Sample.

The 6 years-old children are 38 in Dakar, 9 in Saint-Louis and 49 in Kaolack

The 5 years-old children are 87 in Dakar, 101 in Saint-Louis and 88 in Kaolack; the 4 years-old children are 114 in Dakar, 94 in Saint-Louis and 66 in Kaolack; the 3 years-old children are 60 in Dakar, 98 in Saint-Louis and 97 in Kaolack.

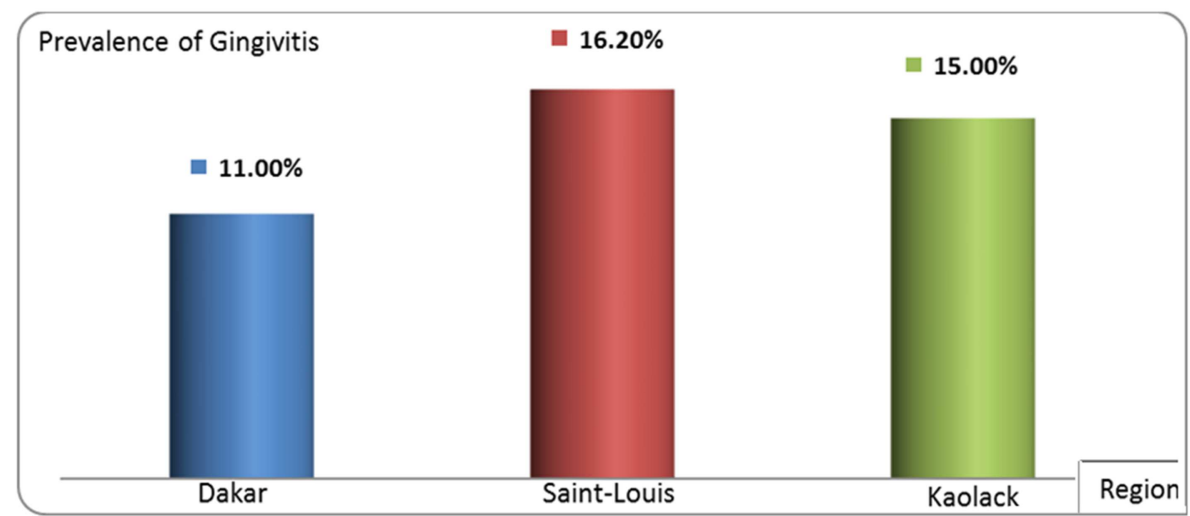

Figure 2. Prevalence of Gingivitis by Region. 
Table 2. Distribution of Gingivitis according to Socio-Demographic data

\begin{tabular}{|c|c|c|c|c|}
\hline Sociodemographic data & Modalities & Frequency & Gingivitis Prevalence & P-Value \\
\hline \multirow{2}{*}{ Sexe } & Male & 391 & $15.3 \%$ & \multirow{2}{*}{0.385} \\
\hline & Female & 510 & $13.1 \%$ & \\
\hline \multirow{3}{*}{ Age in years } & 3 & 255 & $12.9 \%$ & \multirow{3}{*}{0.405} \\
\hline & 4 & 274 & $15.7 \%$ & \\
\hline & 5 & 276 & $15.2 \%$ & \\
\hline \multirow{2}{*}{ Occupation of parents } & Salaried & 205 & $12.2 \%$ & \multirow{2}{*}{0.425} \\
\hline & Non Salaried & 696 & $14.7 \%$ & \\
\hline
\end{tabular}

Table 3. Distribution of Gingivitis according to the Preventive Methods.

\begin{tabular}{llll}
\hline Preventive Methods & & Frequency & Gingivitis Prevalence \\
\hline \multirow{2}{*}{ Brushing Fequency } & $<1 /$ day & 349 & $17.5 \%$ \\
& 1 à +/day & 521 & $12.3 \%$ \\
Visit to the dentist & $\leq 1$ year & 58 & $8.6 \%$ \\
& $>1$ year & 812 & $14.5 \%$ \\
\hline
\end{tabular}

\subsection{Gingival Status and Associated Factors}

Fourteen percent of children had an inflamed gingiva. Table 2 shows the distribution of gingivitis according to socio-demographic data which were not significantly associated with.

Depending on the region, gingivitis is statistically, significantly, and differently distributed $(\mathrm{p}<0.01)$ (Fig 2). Between Dakar and Saint-Louis $(p<0.01)$ and between Dakar and Kaolack ( $\mathrm{p}<0.02)$, the differences were significant. However between Saint-Louis and Kaolack, there was no significant difference ( $p>0.9$ ).

The prevalence of gingivitis varied according to the frequency of brushing and the visit to the dentist as described in Table 3.

Gingivitis is significantly associated with the frequency of brushing and not significantly associated with visiting the dentist.

\section{Discussion}

\subsection{Difficulties and Limitations}

The major difficulty encountered was moving between the boxes toddlers that were distant from each other, especially in the region of Kaolack where the rural area is vast. The second difficulty was encountered in gathering completed questionnaires by parents who often respond with delay. However, given both stratified sampling and cluster sampling, and relatively high levels of completeness $(93 \%$, $91 \%$ and $90 \%$ ), selection biases were minimized; the sample was representative of the boxes toddlers selected in regions. The lack of precision often linked to the cluster sample was reduced for each region, because the based sampling was identified in a homogeneous geographical area. The study had not suffered from information bias on the assessment of gingivitis, because only one person already calibrated to techniques of collect, has collected the information. In contrast, information bias could come from parent reports on prevention methods applied to children.

\subsection{Sociodemographic Data}

During the study, 901 children were examined from which there were 391 boys $(43.4 \%)$ and 510 girls (56.6\%). This situation in preschool does not reflect the distribution of the 0 5 years of the Senegalese population in which there are more boys (50.4\%) than girls [14]. However Cisse [15] et al had found almost the same proportions in a similar study carried out in the region of Dakar (57\% of girls versus $43 \%$ of boys). Similarly Wyne et al [16] had found 52\% of girls in a sample of 789 preschool in Saudi Arabia. On the other hand, Medina et al [17] in a study in Mexico have found $48.3 \%$ of girls. Our sample showed a lower number of 6 years-old children representing $10.7 \%$ (Fig 1). At this age, many children are leaving the preschool level, going to the school level; this may explain the declining enrollment. According to the occupation of parents, $77.3 \%$ were non-salaried including farmers, artisans, merchants, and other low income persons. These nonsalaried parents are considered as low-income people compared to salaried. The introduction of the boxes toddlers goes in the direction of democratizing preschool level, formerly reserved to wealthy urban families [10].

\subsection{Prevalence of Gingivitis and Associated Factors}

Overall, $14.0 \%$ of the sample had gingivitis. The prevalence of gingivitis was high compared to that found in Leroy's study [18] conducted in a sample of 2190 Flemish children (Belgium) which was 4\%. Many surveys have shown that gum disease was widespread among children. The prevalence of gingivitis was much higher and the severity increased more in many countries of Asia and Africa than in the US or the European countries [18-23], despite the methodological approaches that vary from one study to another, particularly in the operational definition of gingivitis. By sex, the rate of children with gingivitis was 
higher among boys (15.3\%) than among girls (13.1\%), but this difference was not significant $(\mathrm{p}=0.38)$. The association was not significant in the gingivitis-age and gingivitisoccupation of parent's relationships (Table 2). However, from one region to another, the prevalence of gingivitis was different (Fig 2; p <0.01). The gingivitis rate found in the region of Dakar was different from the rates found in the regions of Saint-Louis and Kaolack, which were similar. This difference between Dakar and other regions may be due to urbanization and its consequences involving, in Africa, more opportunities for parents (nearest health facilities, more information on available health, etc.) to better take care of the oral health of their children. Studies conducted in India the group of 5 years, and among other age groups, have shown more schoolchildren affected with gingivitis in rural areas than in urban areas [22, 23]. Overall, except for "region" these results suggest a lack of social inequalities in the occurrence of gingivitis for studied variables. However, inequalities can be estimated even with other variables such as educational level of parents and status of social protection, that we have not considered in this study. Gingivitis is often associated with children with poor hygiene, malnutrition [8, $21,24]$ and certain systemic diseases [25]. The prevalence of gingivitis found in Dakar (11.0\%) (Figure 2), is lower than that found by Cisse et al [15] in the same target population which was $33 \%$, so 3 times higher in a study conducted in 2004 and published in 2009; we believe that children had better gingival health 10 years later, probably because of improved nutrition and education in the boxes toddlers, as well as parental involvement. Associations between gingival condition and preventive methods (brushing frequency and visiting the dentist) were respectively significant and not significant (Table 3 ). In addition to education provided in the boxes toddlers, the more educated parents might contribute to the results of the effect of brushing on gingivitis reduction by providing more support due to their good level of information. Although the association between gingivitis and dental visit was not statistically significant, the children paying visits to dentists more often are more likely to receive practitioner advice on compliance with hygienic methods. In a study conducted in Cambodia [26], the authors concluded that the lack of brushing that induces the plate was strongly associated with gingivitis among students ( $p<0.0001)$. Studies conducted in South Africa and Lithuania have shown that, most parents brought their children to the dentist when they are hurt $[24,27]$; this implies that often painless gum disease do not motivate visits to dentists.

\section{Conclusion}

This study on gingivitis conducted in boxes toddlers in 3 regions of Senegal has shown that the presence of gingivitis depended neither on sex, age, or the occupation of the parents, nor on visits to the dentist. However, gingivitis was statistically associated with the region (urban region versus rural or semirural regions) and the frequency of brushing. These results suggest the importance of hygiene and perhaps the oral health literacy in the prevention of this infection often overlooked because painless most of the time. The probable precursor role of gingivitis in the occurrence of noma mutilating affection of the face in children in Africa, should further motivate health officials to develop integrated community programs against gingivitis in boxes toddlers in Senegal.

\section{References}

[1] WHO. World Report on Violence and Health. www.who.int/iris/bitstream/10665/67410/1/a77101_fre.pdf

[2] WHO. Global surveillance of infectious diseases. http://www.who.int/mediacentre/factsheets/fs200/fr/

[3] Abanto J, Carvalho TS, Mendes FM, Wanderley MT, Bönecker M, Raggio DP. Impact of oral diseases and disorders on oral health-related quality of life of preschool children. Community Dent Oral Epidemiol. 2011; 39: 105-114.

[4] Bailleul-Forestier I, Naulin-IFI C. Periodontal child. Encyclical. Med. Chir. Odontologie. 2001; 10: 23-415

[5] Matsson L, Golberg P. Gingival inflammatory reaction in children at different ages. J Clin Periodontol. 1985; 12: 98103.

[6] Adair SM. Dietary counseling time for a nutritionist in the office? Pediatr Dent. 2004; 26 (5): 389.

[7] Wamba. Etiology of noma in Zinder (Niger): socioanthropological study. Tropical Medicine and Health 2013; 23 (3): 287-293.

[8] Diouf M. Cisse D. Faye A. Niang P. Seck I. Faye D. Lo CM. Prevalence of necrotizing ulcerative gingivitis and associated factors in Koranic boarding schools in Senegal. Community Dent Health. 2012; 29 (2): 184-187.

[9] Bassamat. Quality, equity and diversity in preschool: The box toddlers in Senegal. Sevres International Education Review 2010: 53 .

[10] Ministry of Family and Early Childhood. Box toddlers: response to support early childhood. Dakar 2002: 13p

[11] Unesco. Report review of education policy and protection of childhood in Senegal. Breda. Dakar 2009.

[12] WHO. Surveys of Oral Health. Fundamental methods. WHO, Geneva, 1998: 67p.

[13] Colliot C, Boillot A, Bouchard P. Periodontal indices. In: Bouchard P. eds. Periodontology and implant dentistry: Periodontal Medicine. Volume 1, Paris: Edition Lavoisier Medical Sciences, 2015: 682.

[14] National Agency of Statistics and Demography. General Census of Population and Housing. Agriculture and Livestock. 2013: 36p.

[15] Cisse D, Diouf M, Faye D, Lo CM, Sembene NM. Status of dental caries in the toddlers' residences in the region of Dakar. Senegal. Odontostomatol Trop. 2009; 32 (125): 25-31.

[16] Wyne AH, Chohan AN, Jastaniyah N, Al-Khalil R. Bilateral occurrence of dental caries and oral hygiene in preschool children of Riyadh. Saudi Arabia. Odontostomatol Trop. 2008; 31 (124): 19-25. 
[17] Medina-Solís CE, Segovia-Villanueva A, Estrella-Rodríguez R, Maupomé G, Avila-Burgos L, Pérez-Nuñez R. Association between socioeconomic status and oral hygiene among preschoolers enrolled in the IMSS preventive dental program in Campeche. Gac Med Mex. 2006; 142 (5): 363-368.

[18] Leroy R, Jara A, Martens L, Declerck D. Oral hygiene and gingival health in Flemish pre-school children. Community Dent Health. 2011; 28 (1): 75-81.

[19] Peretz B, Machtei EM, Bimstein E. Periodontal status in child hood and early adolescence: three-year follow- up. J Clin Pediatr Dent. 1996; 20: 641-650.

[20] Petersen PE, Jürgensen N. Promoting oral health of children through schools - Results from a WHO global survey 2012. Communit Dent Health. 2013; 30: 204-218.

[21] Azodo CC, Agbor AM. Gingival health and oral hygiene practices of schoolchildren in the North West Region of Cameroon. BMC Res Notes. 2015; 8 (1): 385. doi: 10.1186/s13104-015-1350-2.

[22] Kaur A, Gupta N, Baweja DK, Simratvir M. An epidemiological study to determine the prevalence and risk assessment of gingivitis in 5-12- and 15-year-old children of rural and urban area of Panchkula (Haryana). Indian J Dent Res. 2014; 25 (3): 294-299.

[23] Sharva V, Reddy V, Bhambal A, Agrawal R. Prevalence of Gingivitis among Children of Urban and Rural Areas of Bhopal District. India. J Clin Diagn Res. 20148 (11): ZC52ZC54. Published online 2014 Nov 20. doi: 10.7860/JCDR/2014/10092.5135

[24] Gordon N. Oral health care for children attending a malnutrition clinic in South Africa. Int J Dent Hyg. 2007; 5 (3): $180-186$.

[25] Daković D, Mileusnić I, Hajduković Z, Čakić S, HadžiMihajlović M. Gingivitis and periodontitis in children and adolescents suffering from type 1 diabetes mellitus. Vojnosanit Pregl. 2015; 72 (3): 265-273.

[26] Shidara EK, McGlothlin JD, Kobayashi S. A vicious cycle in the oral health status of schoolchildren in a primary school in rural Cambodia. Int J Dent Hyg. 2007; 5 (3): 165-173.

[27] Vaitkeviciene V, Milciuviene S, Zaborskis A. Oral hygiene of preschool children in Kaunas city and their parents' attitude towards children's oral health. Medicina (Kaunas). 2005; 41 (5): 427-434. 